\title{
Global stability of an HIV/AIDS model with infection age and impulsive persuasion rate
}

\author{
Xiaoyan Wang ${ }^{1}$, Junyuan Yang ${ }^{1,2 \star}$ and Xuezhi $\mathrm{Li}^{3}$ \\ ${ }^{1}$ Department of Applied Mathematics, Yuncheng University, Yuncheng Shanxi 044000 P. R. China. \\ ${ }^{2}$ Beijing Institute of Information and Control, Beijing Beijing 100037 P. R. China. \\ ${ }^{3}$ Department of Mathematics, Xinyang Normal University, Xinyang Hennan 464000 P. R. China.
}

Accepted 8 September, 2011

\begin{abstract}
In this paper, an impulsive persuasion strategy to eradicate HIV/AIDS is studied. Since infection age is an important factor of HIV progression, we incorporate the infection age into the model. Using integral equation theory and operator semi group theory, we analyze the dynamical behaviors of this model and point out that there exists an infection-free periodic solution which is globally asymptotically stable if $R_{0}<1$. This condition depends on impulsive persuasion proportion $p_{n}$ and inter impulsive time $\tau$.
\end{abstract}

Key words: Human immunodeficiency virus/acquired immunodeficiency syndrome model, persuasion rate, global attractor.

\section{INTRODUCTION}

During the past two decades, human immunodeficiency virus (HIV) disease has become one of the major public problems in the world. In 2007, advances in the methodology of estimations of HIV epidemics applied to an expanded range of country data have resulted in substantial changes in estimates of numbers of people living with HIV worldwide (Aggarwala, 2006; Report, 2007). In 2007, the Ministry of Health, UNAIDS and WHO prepared an updated assessment of the AIDS epidemic in China. The estimation results showed by the end of 2007 approximately 700,000 are HIV positive (range 550,000 to 850,000 ). Currently, China's HIV epidemic remains one of low prevalence overall, but with pockets of high infection among specific sub-populations and in some localities. The characteristic of the epidemic in China are: the epidemic continues to expand, but the rate is slowing; sexual transmission is now the main mode for the spread of HIV; geographic distribution is highly varied; and the epidemic continues to be driven by highrisk behavior within particular sub-populations (AIDS, 2007). From a theoretical point of view, the HIV/AIDS

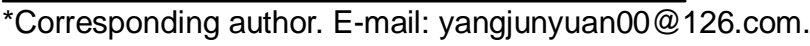

epidemic provides a large number of new problems to mathematicians, biologists and epidemiologists, since it has a lot of features different from traditional infectious diseases. Hence, the study of HIV/AIDS has stimulated the recent development of mathematical epidemiology. In the following, we briefly discuss the characteristics which should be taken into account in mathematical models for HIV dynamics. According to a recent statistical analysis on acquired immunodeficiency syndrome (AIDS) awareness among married couples in Bangladesh (Rahman and Rahman, 2007), media and education play a tremendous role in mounting AIDS awareness among the residents. According to Rahman and Rahman (2007), both women and men who regularly watch TV were 8.6 times more likely to be aware about AIDS compared to those who never watch TV. In addition, CDC governments produced much propaganda about AIDS knowledge which can persuade many people reduce times of risky behavior.

In developing and undeveloped countries, drug-fast for HIV/AIDS is highly cost, categories of medicine goes short and people lack knowledge about HIV/AIDS. One of the best measures is to tell people the correct preventive knowledge of the disease as soon as possible through media and education. However, it is reasonable to study 
theoretically the application of pulse persuasion in the control of HIV/AIDS. Many scholars have studied pulse vaccination and treatment strategies in HIV models (Krakovska and Wahl, 2007; Liu et al., 2006; Smith and Wahl, 2004, 2005). But the persuasion is rarely in recently epidemic models. As the authors have known, Thripathi et al. (2007) proposed a nonlinear model to study the effect of screening of unaware infective on the spread of HIV/AIDS in a homogenous population with constant immigration of infective. They had shown that screening of unaware infective had the effect of reducing the spread of AIDS epidemic. Infection age (Liu et al., 2006,2008 ) is an important feature of many slowly progressing diseases like HIV. The disease has a long latency period. In HIV, the fast initial phase appears to be followed by a long phase with low virus titers. Therefore, it is reasonable to consider infection age (the time lapsed since infection) in disease progression. We introduce infection age and impulsive persuasion into our model.

This paper is organized as follows: subsequently, it introduces an HIV/AIDS model with infection age and pulse persuasion, thereafter, the global attractor of the infection-free periodic solution is studied by using the spectral theory and comparison principle.

\section{THE MODEL FORMULATION}

Here, we introduce an HIV/AIDS model with pulse persuasion and infection age. We consider a population whose death rate is $\mu$, and the input rate is $\Lambda$. The host population is divided into three groups: susceptible, infected and those with AIDS. The susceptible population is persuaded at time $n \tau$ every $\tau$ months; $\tau$ is the period of pulse persuasion, $n \tau$ is the time at which we apply the $n t h\left(n \in N_{+}\right)$pulse, $n \tau^{-}$is the time just before applying the $n t h$ pulse, a fraction $p_{n}$ of the susceptible having risk behavior are persuaded, where $p_{n}$ is a period function, that is: $(n+q) \tau=n \tau+T, p_{n+q}=p_{n}$. In addition, $[0 \quad T] \cap\{\tau\}\{, 2 \tau, \cdots, q \tau\}$. This assumption is realistic in reality. Simple persuasion may be effective in first period when they know the ponderance of HIV/AIDS. However, risk group may be declined to receiving the simple persuasion way; we should change the styles of persuasion which tends to be the same effect in the second period. The susceptible can be infected by infected class $I(a, t)$ at a transmission rate $\beta(a)$ and go to the infected class. We call the time individuals spent in the infected class infection age $a$. Infected individuals can develop those with AIDS at a transmission rate $\alpha(a) . d$ is the death rate due to AIDS. We obtain the following system:

$$
\left\{\begin{array}{l}
\frac{d S}{d t}=\Lambda-\mu S-S \int_{0}^{\infty} \beta(a) I(a, t) d a, t \neq n \tau, \\
S(n \tau)=\left(1-p_{n}\right) S\left(n \tau^{-}\right), n \in N_{+}, \\
\frac{\partial I(a, t)}{\partial a}+\frac{\partial I(a, t)}{\partial t}=-(\mu+\alpha(a)) I, 0<a<\infty, t \neq n \tau, \\
I(0, t)=S(t) \int_{0}^{\infty} \beta(a) I(a, t) d a, 0 \leq a<\infty, \\
I(a, n \tau)=D(a) I\left(a, n \tau^{-}\right), 0 \leq \tau<\infty, \\
\frac{d A}{d t}=\int_{0}^{\infty} \alpha(a) I(a, t) d a-\mu A-d A, t \neq n \tau, \\
A(n \tau)=A\left(n \tau^{-}\right), n \in N_{+} .
\end{array}\right.
$$

With the initial conditions:

$S(0)=S_{0} \geq 0, \quad I(a, 0)=I_{0}(a) \geq 0, \quad A(0)=A_{0} \geq 0$,

Where:

$$
N=S(t)+\int_{0}^{\infty} I(a, t) d \tau+A(t)
$$

The initial density $I_{0}(a)$ is assumed to be integrable and compactly supported in

$$
[0, \infty), \beta(a), \alpha(a) \in L^{\infty}(0, \infty) D(a)=\left\{\begin{array}{l}
1, a>0, \\
1-p_{n}, a=0 .
\end{array}\right.
$$

If system of Equation 1 has a solution $(S(t), I(a, t), A(t))$, then $S(t), I(a, t), A(t)$ are discontinuous at $n \tau, n \in N_{+}$, and $n \tau$ is a point of discontinuity of the first kind. So, we may assume that $S(t), I(a, t), A(t)$ are right continuous at $n \tau$. We can obtain that $S(t), A(t)$ are continuous in the interval $[n \tau,(n+1) \tau), \quad I(a, t)$ is continuous for $a>0, t \in[n \tau,(n+1) \tau)$.

\section{Lemma 1}

Suppose $f:[0, \infty) \rightarrow R$ is a bounded function Lemma1, then:

$$
\limsup _{t \rightarrow \infty}\left|\int_{0}^{t} k(\theta) f(t-\theta) d \theta\right| \leq|f|^{\infty} k_{L(0, \infty)}
$$

\section{Lemma 2}

Let the function $w \in P C^{\prime}([0, \infty), R)$ satisfies the 
inequalities (Lakshmikantham et al., 1989):

$$
\left\{\begin{array}{l}
\dot{w}(t) \leq f(t) w(t)+g(t), t \neq n \tau, t>0 \\
w(n \tau) \leq f_{n} w\left(n \tau^{-}\right)+g_{n}, n \in N_{+}, \\
w(0) \leq w_{0} .
\end{array}\right.
$$

Where $f(t), g(t) \in P C([0, \infty), R), f_{n}>0, g_{n}$ and $w_{0}$ are constants. Then for.

$$
\begin{aligned}
& t>0 \\
& w(t) \leq w(0) \prod_{0<n \tau<t} f_{n} \exp \left(\int_{0}^{t} f(s) d s\right)+\int_{0}^{t} \prod_{s \leq n \tau<t} f_{n} \exp \left(\int_{0}^{t} f(r) d r\right) g(s) d s \\
& \quad+\sum_{0<n \tau<t} \prod_{n \tau \leq j \tau<t} \int_{0}^{t} f_{j} \exp \left(\int_{n \tau}^{t} f(s) d s\right) g_{n} .
\end{aligned}
$$

Analogously,

$$
\begin{aligned}
w(t) & \geq w(0) \prod_{0<n<t} f_{n} \exp \left(\int_{0}^{t} f(s) d s\right)+\int_{0}^{t} \prod_{s \leq n \tau<t} f_{n} \exp \left(\int_{0}^{t} f(r) d r\right) g(s) d s \\
& +\sum_{0<n \tau<t} \prod_{n \tau \leq j j<<t} \int_{0}^{t} f_{j} \exp \left(\int_{n \tau}^{t} f(s) d s\right) g_{n} .
\end{aligned}
$$

For all $t \leq 0$ if all the inequalities of Equation 2 are inverse.

\section{THE ATTRACTOR OF THE EQUILIBRIUM}

We first demonstrate the existence of an infection-free solution in which infection individuals are entirely absent from the population permanently, that is $I(a, t)=0, t \geq 0$. Under this condition, the susceptible must satisfy:

$$
\left\{\begin{array}{l}
\frac{d S}{d t}=\Lambda-\mu S, t=n \tau, \\
S(n \tau)=\left(1-p_{n}\right) S\left(n \tau^{-}\right), n \in N_{+} .
\end{array}\right.
$$

\section{Theorem 1}

System 1 always has a positive periodic solution $E^{*}=\left(S^{*}, 0,0\right)$.

\section{Proof 1}

We consider the linear non-homogeneous impulsive equations:

$$
\left\{\begin{array}{l}
\frac{d S}{d t}=\Lambda-\mu S, t=n \tau, \\
S(n \tau)=\left(1-p_{n}\right) S\left(n \tau^{-}\right), n \in N_{+} .
\end{array}\right.
$$

Let:

$W(t, s)=\prod_{s \leq n \tau<t}\left(1-p_{n}\right) e^{-\mu(t-s)}$

be the Cauchy matrix for the respective homogeneous equation. Then:

$S(t)=W(t, 0) S(0)+\int_{0}^{t} \Lambda W(t, s) d s$

is a solution of Equation (3). If this solution is T-periodic, that is $S(0)=S(T)$ and then:

$$
(1-W(T, 0)) S(0)=\int_{0}^{T} \Lambda W(T, s) d s .
$$

Since the multiplier $W(T, 0)$ of the homogeneous equation:

$$
\left\{\begin{array}{l}
\frac{d x}{d t}=-\mu x, t=n \tau, \\
x(n \tau)=\left(1-p_{n}\right) x\left(n \tau^{-}\right), n \in N_{+} .
\end{array}\right.
$$

is always less than 1 and $\int_{0}^{T} \Lambda W(T, s) d s>0$. Equation (4) has a unique solution $S(0)$. To the initial value $S(0)$, we obtain there exists corresponds the unique $T_{-}$ periodic solution of (3). Denote this solution by $S^{*}(t)$.

\section{Lemma 3}

If $\alpha(a), \beta(a) \in L^{\infty}(0, \infty)$ (Liu et al., 2008), then Equation (1) has a unique nonnegative solution $(S(t), I(a, t), A(t)) \in P C\left(R_{+}, R\right) \times P C\left(R_{+}, L^{1}([0, \infty))\right.$ $\left.\times P C\left(R_{+}, R\right)\right)$ with respect to initial data $\left(S_{0}, I_{0}(a), A_{0}\right) \in \Omega_{0}=\left(S_{0}, I_{0}(a), A_{0}\right) \mid S_{0} \in R_{+}, I_{0}(a)$ is integrable and supported in $\left.[0, \infty), A_{0} \in R_{+}, S_{0}+\int_{0}^{\infty} I_{0}(a) d a+A_{0} \leq \frac{\Lambda}{\mu}\right\}$

We note that the system: 


$$
\left\{\begin{array}{l}
\frac{d S_{n}}{d t}=\mu-\mu S_{n}-S_{n}(t) \int_{0}^{\infty} \beta(a) I_{n}(a, t) d a, n \tau<t<\infty \\
\frac{\partial I_{n}}{\partial t}+\frac{\partial I_{n}}{\partial a}=-\mu I_{n}-\alpha(a) I_{n}, 0<a<\infty \\
I_{n}(0, t)=S_{n}(t) \int_{0}^{\infty} \beta(a) I_{n}(t, a) d \tau, n \tau \leq t<\infty \\
\frac{d A_{n}}{d t}=\int_{0}^{\infty} \alpha(a) I_{n} d a-\mu A_{n}-d A_{n}, 0<a<\infty
\end{array}\right.
$$

With initial conditions:

$n=0, S_{0}(0)=S_{0} \geq 0, I_{0}(a, 0)=I_{0}(a) \geq 0, A_{0}(0)=A_{0} \geq 0 ; n \in N_{+}, S_{n}(n \tau)=\left(1-p_{n}\right) S_{n-1}\left(n \tau^{-}\right)$, $I_{n}(a, n \tau)=D(a) I_{n-1}\left(a, n \tau^{-}\right), A_{n}(n \tau)=A_{n-1}\left(n \tau^{-}\right)$has a unique nonnegative continuous solution (Webb, 1985; lannelli, 1995).

Let $\left(S_{n}(t), I_{n}(a, t), A_{n}(t)\right)$,

$t \in[n \tau, \infty), \quad \tau \in(0, \infty), n \in N$ be the solution of the system of Equation (5). Then we have the representation:

$(S(t), I(a, t), A(t))=\left(S_{n}(t), I_{n}(a, t), A_{n}(t)\right), a \geq 0, t \in[n \tau,(n+1) \tau, n=0,1,2, \ldots$.

If $t \in[n \tau,(n+1) \tau)$, in Equation 1, for sufficiently large $t$, we obtain

$\lim \sup S(t) \leq S^{*}(T)$

Let:

$$
\left\{\begin{array}{l}
Z(t)=S(t) \int_{0}^{\infty} \beta(a) I(a, t) d a, t \in[0, \infty), \\
Z_{n}(t)=S_{n}(t) \int_{0}^{\infty} \beta(a) I_{n}(a, t) d a, t \in[n \tau, \infty), n=1,2, \ldots . .
\end{array}\right.
$$

Furthermore, integrating the third differential equation in Equation 1 along the characteristic line $t-a=$ constant, for $t \in[0, \tau)$, we get the following formula:

$i_{0}(a, t)=\left\{\begin{array}{l}Z_{0}(t-a) K_{0}(a), t \geq a, \\ D(a-t) i_{0}(a-t) \frac{K_{0}(a)}{K_{0}(a-t)}, t<a,\end{array}\right.$

When $t \in[n \tau,(n+1) \tau)$,

$i_{n}(a, t)=\left\{\begin{array}{l}Z_{n}(t-a) k_{0}(a), t-n \tau \geq a, \\ D(n \tau+a-t) i_{n-1}(n \tau+a-t) \frac{K_{0}(a)}{K_{0}(a-t)}, t-n \tau<a .\end{array}\right.$

Hence, we have:

$$
\begin{aligned}
Z_{n}(t)= & S_{n}(t) \int_{0}^{t-n \tau} \beta(a) Z_{n}(t-a) k_{0}(a) d a+S_{n}(t) \int_{t-n \tau}^{\infty} D(n \tau+a-t) \\
& \beta(a) i_{n-1}(n \tau+a-t) \frac{K_{0}(a)}{K_{0}(n \tau+a-t)} d a \\
& \leq S^{*} \int_{0}^{t-n \tau} \beta(a) Z_{n}(t-a) k_{0}(a) d a+S^{*} \int_{t-n \tau}^{\infty} \beta(a) i_{n-1}(n \tau+a-t) d a \\
& \leq S^{*} \int_{0}^{t-n \tau} \beta(a) Z_{n}(t-a) k_{0}(a) d a+S^{*} \int_{t-n T}^{t-(n-1) \tau} \beta(a) Z_{n-1}(t-a) K_{0}(a) d a \\
& +S^{*} \int_{t-(n-1) \tau}^{\infty} \beta(a) i_{n-1}(n \tau+a-t) \frac{K_{0}(a)}{K_{0}(n \tau+a-t)} d a \\
& \leq S^{*} \int_{0}^{t-n \tau} \beta(a) Z_{n}(t-a) k_{0}(a) d a+S^{*} \int_{t-n \tau}^{t-(n-1) \tau} \beta(a) Z_{n-1}(t-a) K_{0}(a) d a+\ldots
\end{aligned}
$$

$+S^{*} \int_{t-\tau}^{t} \beta(a) Z_{0}(t-a) K_{0}(a) d a+S^{*} \int_{t}^{\infty} D(a-t) \beta(a) i_{0}(a-t) \frac{K_{0}(a)}{K_{0}(a-t)} d a$

$\leq S^{*} \int_{n \tau}^{t} \beta(t-a) Z_{n}(a) K_{0}(a) d a+S^{*} \int_{(n-1) \tau}^{n \tau} \beta(t-a) Z_{n-1}(a) K_{0}(a) d a+\ldots$

$+S^{*} \int_{\tau}^{0} \beta(t-a) Z_{0}(a) K_{0}(a) d \tau+S^{*} \int_{t}^{\infty} D(a) \beta(t-a) i_{0}(\tau) \frac{K_{0}(\tau)}{K_{0}(a-t)} d a$

$=S^{*} \int_{n \tau}^{t} \beta(t-a) Z(a) k_{0}(a) d a+S^{*} \int_{(n-1) \tau}^{n \tau} \beta(t-a) Z(a) k_{0}(a) d a+\cdots+F(t)$.

For $t \in[n \tau,(n+1) \tau)$, we obtain:

$$
Z(t)=Z_{n}(t) \leq S^{*}(T) \int_{0}^{t} \beta(a) Z(t-a) k_{0}(a) d a+F(t),
$$

where

$K_{0}(a)=e^{-\mu a-\int_{0}^{a} \alpha(\xi) d \xi}, F(t)=S_{n}(t) \int_{t}^{\infty} \beta(a) I_{0}(a-t) \frac{K_{0}(a)}{K_{0}(a-t)} d a$, and $\lim _{t \rightarrow \infty} F(t)=0$.

From Equations (6) and (9), we have:

$Z^{\infty} \leq R_{0} Z^{\infty}$

Where $_{Z^{\infty}}=\limsup Z(t), R_{0}=S^{*}(T) \int_{0}^{\infty} \beta(a) K_{0}(a) d a$. If

$R_{0}<1$ holds, we have $Z^{\infty}=0$.

Therefore, the following proposition holds.

\section{Proposition 1}

Suppose $R_{0}<1$, then $\lim _{t \rightarrow \infty} Z(t)=0$.

Let

$I(t)=\int_{0}^{\infty} I(a, t) d a, Z(t)=S(t) \int_{0}^{\infty} \beta(a) I(a, t) d a, t \in[0, \infty)$.

As the proof aforementioned, we get:

$I(t) \leq \int_{0}^{t} Z(t-a) K_{0}(a) d a+G(t)$, 
Where $\quad G(t)=\int_{0}^{\infty} I_{0}(a-t) \frac{K_{0}(a)}{K_{0}(a-t)} d a, \lim _{t \rightarrow \infty} G(t)=0$.

Using

Proposition 2, if:

$R_{0}=S^{*}(T) \int_{0}^{\infty} \beta(a) K_{0}(a) d a<1$,

We have $I^{\infty} \leq \int_{0}^{\infty} K_{0}(a) d a Z^{\infty}=0$. Thus, it holds that:

$\lim _{t \rightarrow \infty} \int_{0}^{\infty} I(a, t) d a=0$.

Next, we show that $S(t)-S^{*}(t) \rightarrow 0$ as $t \rightarrow \infty$ holds. According to Equation 1 and Lemma 2, we get:

$S-S^{*}=\left(S_{0}-S^{*}(0)\right) \prod_{0<1<<1}\left(1-p_{n}\right) e^{-\mu t}-\int_{0}^{t} \prod_{S \leq n<\varepsilon<t}\left(1-p_{n}\right) e^{-\mu(t-s)} S(s) \int_{0}^{\infty} \beta(a) I(a, t) d a d s$,

and

$\lim _{t \rightarrow \infty}\left(S_{0}-S^{*}(0)\right) \prod_{0<n \tau<t}\left(1-p_{n}\right) e^{-\mu t}=0$.

From Equation 10, it is easy to get:

$\lim _{t \rightarrow \infty}\left[S(t)-S^{*}(t)\right]=0$.

From Equations 1 and 10, it is easy to get:

$A=A_{0} e^{-(\mu+d) t}+\int_{0}^{t} e^{-(\mu+d)(t-s)} \int_{0}^{\infty} \alpha(a) I(a, t) d a d s$.

It is easy to obtain $A \rightarrow 0$, as $t \rightarrow \infty$.

From the aforementioned, we have the following results:

\section{Theorem 2}

If $\beta(a), \alpha(a) \in L^{\infty}[0, \infty)$ and $R_{0}<1$ holds, then the periodic infection-free $E^{*}$ of system of Equation 1 is a global attractor. In order to investigate the locally asymptotically stability of the periodic infection-free solution $\left(S^{*}, 0,0\right)$ for system of Equation 1 , we first rewrite Equation 1 and consider the equivalent system:

\footnotetext{
$\left\{\begin{array}{l}\frac{d S}{d t}=\Lambda-\mu S-S \int_{0}^{\infty} \beta(a) I(a, t) d \tau, t \neq n \tau, \\ S(n \tau)=\left(1-p_{n}\right) S\left(n \tau^{-}\right), n \in N_{+}, \\ \frac{\partial I(a, t)}{\partial t}+\frac{\partial I(a, t)}{\partial a}=-\beta(a) I(a, t) S(t)-(\mu+\alpha(a)) I(a, t), 0<a<\infty, t \neq n \tau, \\ I(0, t)=0,0<a<\infty, t \neq n \tau, \\ I(a, n \tau)=D(a) I\left(a, n \tau^{-}\right), 0<a<\infty, \\ \frac{d A}{d t}=\int_{0}^{\infty} \alpha(a) I(a, t) d a-\mu A-d A, t \neq n \tau, \\ A(n \tau)=A\left(n \tau^{-}\right), n \in N_{+},\end{array}\right.$
}

With the initial conditions:

$S(0)=S_{0} \geq 0, I(a, 0)=I_{0}(a), A(0)=A_{0}$,

Let $S(t)=s(t)+S^{*}, I(a, t)=i(a, t), A(t)=a(t), \quad$ from Equation 11, we have:

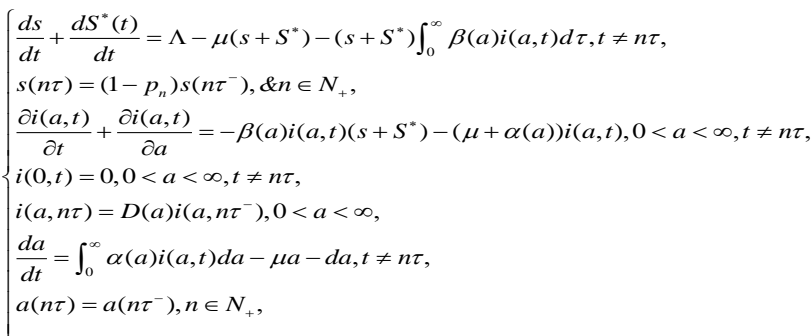

It is easy to see that linearization of the system of Equation 12 at $(s, i, a)=(0,0,0)$ leads to the following system:

$\left\{\begin{array}{l}\frac{d s}{d t}=-\mu s-S^{*} \int_{0}^{\infty} \beta(a) i(a, t) d \tau, t \neq n \tau \\ s(n \tau)=\left(1-p_{n}\right) s\left(n \tau^{-}\right), n \in N_{+}, \\ \frac{\partial i(a, t)}{\partial t}+\frac{\partial i(a, t)}{\partial a}=-\beta(a) i(a, t) S^{*}-(\mu+\alpha(a)) i(a, t), 0<a<\infty, t \neq n \tau \\ i(0, t)=0,0<a<\infty, t \neq n \tau \\ i(a, n \tau)=D(a) i\left(a, n \tau^{-}\right), 0<a<\infty \\ \frac{d a}{d t}=\int_{0}^{\infty} \alpha(a) i(a, t) d a-\mu a-d a, t \neq n \tau \\ a(n \tau)=a\left(n \tau^{-}\right), n \in N_{+}\end{array}\right.$

We can obtain that the system of Equation 13 has a nonnegative solution for initial conditions $s(0)=s_{0} \geq 0, i(a, 0)=i_{0}(a) \geq 0, a(0) \geq 0$. Now we consider a comparable system of system of Equation 13:

$\left\{\begin{array}{l}\frac{d \bar{s}}{d t}=-\mu \bar{s}-S^{*}(T) \int_{0}^{\infty} \beta(a) \bar{i}(a, t) d a, 0<t<\infty, \\ \frac{\partial \bar{i}(a, t)}{\partial a}+\frac{\partial \bar{i}(a, t)}{\partial t}=S^{*}(T) \int_{0}^{\infty} \beta(a) \bar{i}(a, t) d a-(\mu+\alpha(a)) \bar{i}(a, t) 0<a<\infty, 0<t<\infty, \\ \frac{d \bar{a}}{d t}=\int_{0}^{\infty} \alpha(a) \bar{i}(a, t) d a-\mu \bar{a}-d \bar{a}, 0<t<\infty .\end{array}\right.$

With boundary condition $\bar{i}(0, t)=0$, and initial conditions $\bar{s}(0)=s_{0}, \bar{i}(a, 0)=i_{0}(a), \bar{a}(0)=a_{0}$. System of Equation 14 has a unique nonnegative solution $\bar{s}(t), \bar{i}(a, t), \bar{a}(t)$ and:

$0 \leq i(a, t) \leq \bar{i}(a, t), 0 \leq a(t) \leq \bar{a}(t)$

So if we prove that the system of Equation 14 is stable at $(0,0,0)$, then the system of Equation 13 is stable at 
$(0,0,0)$. We rewrite the system of Equation 14 in the following form:

$$
\left\{\begin{array}{l}
\frac{d \varphi}{d t}=A \varphi(t)+B \varphi(t), \\
\varphi(t)=\left(\varphi_{1}(t), \varphi_{2}(t), \varphi_{3}(t)\right)^{\prime} \\
\varphi(0)=\varphi_{0}
\end{array}\right.
$$

Where $A: D(A) \rightarrow X, X=R \times L[0, \infty) \times R$,

$$
\begin{aligned}
& \left.D(A)=\left(\varphi_{1}, \varphi_{2}(a), \varphi_{3}\right)^{\prime}, \varphi_{2} \in W^{\prime}\left(0, r_{m}\right), \varphi_{1}, \varphi_{3} \in R\right\} \\
& A=\left(-\mu \varphi_{1}-\frac{d \varphi_{2}}{d \tau}-\mu \varphi_{2}-\alpha(a) \varphi_{2}-\mu \varphi_{3}-d \varphi_{3}\right) \\
& B=\left(-S^{*}(T) \int_{0}^{\infty} \beta(a) \varphi_{2}(a) d a S^{*}(T) \int_{0}^{\infty} \beta(a) \varphi_{2}(a) d a \int_{0}^{\infty} \alpha(a) \varphi_{2}(a) d a\right) .
\end{aligned}
$$

Clearly, $B$ is a bounded perturbation of $A$. Moreover, $B$ is compact. Next, we consider the Eigen value problem for the linear system of Equation 16:

$$
\left\{\begin{array}{l}
(\lambda+\mu) \varphi_{1}=-S^{*}(T) \int_{0}^{\infty} \beta(a) \varphi_{2}(a) d \tau=-P_{1}\left(\varphi_{2}\right), \\
\frac{d \varphi_{2}}{d a}+(\lambda+\mu+\alpha(a)) \varphi_{2}=P_{1}\left(\varphi_{2}\right), \\
(\lambda+\mu) \varphi_{3}=P_{2}\left(\varphi_{2}\right)
\end{array}\right.
$$

where:

$$
P_{1}\left(\varphi_{2}\right)=S^{*}(T) \int_{0}^{\infty} \beta(a) \varphi_{2}(a) d a, P_{2}\left(\varphi_{2}\right)=\int_{0}^{\infty} \alpha(a) \varphi_{2}(a) d a .
$$

From Equation 17, we obtain that the characteristic equation has two roots with negative real part, one is $\lambda_{1}=-\mu$, the other is $\lambda_{2}=-(\mu+d)$. Another characteristic root is determined by Equation 19:

$$
S^{*}(T) \int_{0}^{\infty} \beta(a) \int_{0}^{a} e^{-(\lambda+\mu)(a-s)-\int_{0}^{a} \alpha(\xi) d \xi} d s d a=1
$$

We denote the left-hand side in Equation 19 by $\Phi(\lambda)$. If $\lambda \geq-\mu$ is real, then $\Phi(\lambda)$ is decreasing and $\lim _{\lambda \rightarrow \infty} \Phi(\lambda)=0$. In addition, for any $\lambda$ with $\operatorname{Re} \lambda \geq 0$, we have $\Phi(\lambda) \leq \Phi(\operatorname{Re} \lambda)$. Let:

$$
\begin{aligned}
R_{0}^{1} & =S^{*}(T) \int_{0}^{\infty} \beta(a) \int_{0}^{a} e^{-\mu(a-s)-\int_{0}^{a} \alpha(\xi) d \xi} d s d a \\
& =S^{*}(T) \int_{0}^{\infty} \beta(a) \int_{0}^{a} K_{0}(a) e^{\mu s} d s d a \\
& =\frac{S^{*}(T)}{\mu} \int_{0}^{\infty} \beta(a) K_{0}(a)\left(e^{\mu a}-1\right) d a \\
& =\frac{S^{*}(T)}{\mu}\left[\int_{0}^{\infty} \beta(a) K_{0}(a) e^{\mu a} d a-\int_{0}^{\infty} \beta(a) K_{0}(a) d a\right]
\end{aligned}
$$

If $R_{0}^{1}<1$, and if we assume that $\lambda$ is a solution of Equation 19 with $\operatorname{Re} \lambda \geq 0$, we have:

$$
1=|\Phi(\lambda)| \leq \Phi(\operatorname{Re} \lambda) \leq \Phi(0)=R_{0}^{1}<1
$$

Which is contradiction stemming from the assumption. So, we obtain that the solution of Equation 17 has negative real part. We get Proposition 2.

\section{Proposition 2}

If $R_{0}^{1}<1$, the Eigen values of $A+B$ have negative real part. It is easy to get Proposition 3 .

\section{Proposition 3}

The operator $A$ is a closed linear operator and satisfies:

$$
(\lambda-A)^{-n} \leq \frac{1}{(\lambda+\mu)^{n}}
$$

For $\lambda>-\mu$, and all positive integers $n$.

\section{Proof 2}

For any $f=\left(f_{1}, f_{2}, f_{3}\right)^{T} \in X_{+}$, we solve the solution $(\lambda-A) \varphi=f$, and obtain:

$0=-(\lambda+\mu) \varphi_{1}+f_{1}$,

$\frac{d \varphi_{2}}{d a}=-(\lambda+\mu+\alpha(a)) \varphi_{2}+f_{2}$,

$0=-(\lambda+\mu+d) \varphi_{3}+f_{3}$,

$\varphi_{1}(0)=0, \varphi_{2}(0)=0, \varphi_{3}(0)=0$.

From Equation 23, we get: 
$\varphi_{1}=\frac{\varphi_{1}}{\lambda+\mu}$

$\varphi_{2}(a)=e^{-\int_{0}^{a}(\lambda+\mu+\alpha(a)) d a} \int_{0}^{a} f_{2}(\tau) e^{\int_{0}^{\tau}(\lambda+\mu+\alpha(\xi)) d \xi} d \tau$,

$\varphi_{3}=\frac{f_{3}}{\lambda+d+\mu}$.

Then,

$$
\begin{aligned}
\varphi & \leq \frac{\left|f_{1}\right|}{|\lambda+\mu|}+\int_{0}^{\infty} e^{-\int_{0}^{a}(\lambda+\mu+\alpha(a)) d a} \int_{0}^{a}\left|f_{2}(\tau)\right| e^{\int_{0}^{\tau}(\lambda+\mu+\alpha(\xi)) d \xi} d \tau d a+\frac{\left|f_{3}\right|}{|\lambda+\mu+d|} \\
& \leq \frac{f_{1}}{|\lambda+\mu|}+\frac{\int_{0}^{\infty}\left|f_{2}(a)\right| d a}{\left|\lambda+\mu+\alpha_{\infty}\right|}+\frac{\left|f_{3}\right|}{|\lambda+\mu+d|} \\
& \leq \frac{1}{\lambda+\mu}\left(f_{1}+f_{2}+f_{3}\right),
\end{aligned}
$$

Where $f_{\infty}=\inf _{t \rightarrow \infty} f(t)$, then:

$(\lambda-A)^{-n} \leq \frac{1}{(\lambda+\mu)^{n}}$,

For $\lambda>-\varepsilon=\mu$, and all positive integers $n$.

To prove that system of Equation 16 is stable at $(0,0,0)$, we need the following definition and lemma (Webb, 1985).

\section{Definition 1}

A $C_{0}$-Semi group $T(t)$ is called quasi-compact if $T(t)=L(t)+G(t)$ with operator families $L(t)$ and $G(t)$ for $t \geq 0$, such that $\lim _{t \rightarrow \infty} L(t)=0$, and $G(t)$ is eventually compact.

\section{Lemma 4}

Let $H(t)$ be a quasi-compact $C_{0}-$ Semi group and $E$ is an infinitesimal generator. Then $\lim _{t \rightarrow \infty} e^{\varepsilon t} H(t)=0$ for some $\varepsilon>0$, if and only if all Eigen values of $E$ have strictly negative real part. Now we prove the following lemma.

\section{Lemma 5}

Let $T(t)$ be a $C_{0}-$ Semi group and $A+B$ be its infinitesimal generator. If $R_{0}^{1}<1$, then the following inequality holds $w_{0}(A+B) \leq-\mu$, where $w_{0}(A+B)$ denotes the growth of the semi group $T(t), t>0$. If $w(A+B)<0$, the equilibrium $\varphi=0$ of the system of Equation 14 is locally exponentially asymptotically stable in the sense that there exists $\varepsilon>0, M \geq 1$, and $\mu<0$ such that if $\varphi_{0} \in X$ and $\varphi_{0} \leq \varepsilon$, then the solution $\varphi\left(t, t_{0}\right)$ exists globally and $\varphi\left(t, \varphi_{0}\right) \leq M e^{\mu t} \varphi_{0}$ for all $t \geq 0$ (Webb, 1985). This implies that the equilibrium $\varphi=0$ of the system of Equation 11) is locally asymptotically stable. From the aforementioned argument, and Equation 16, we obtain that system of Equation 11 is locally exponentially asymptotically stable at $(0,0,0)$. So we arrive at the following result.

\section{Theorem 3}

If $\beta(\tau), \alpha(\tau) \in L^{\infty}(0, \infty), R_{0}^{1}<1$, and $i_{0}(\tau), \bar{a}_{0}$ are small enough, then $\lim _{t \rightarrow \infty} \bar{i}(t, \tau)=0, \lim _{t \rightarrow \infty} \bar{a}(t)=0$. As for $s$, from the first and second equations of Equation 13 and Lemma 2:

$$
\begin{aligned}
s(t) & =s_{0} \prod_{0<n<t}\left(1-p_{n}\right) e^{-\mu t}-\int_{0}^{t} \prod_{s<n<t}\left(1-p_{n}\right) e^{-\mu(t-s)} S^{*}(s) \int_{0}^{\infty} \beta(a) i(a, t) d a d s \\
& \leq s_{0} \prod_{0<n \tau t}\left(1-p_{n}\right) e^{-\mu t}+\bar{\beta} \int_{0}^{t} \prod_{s<n<\varangle t}\left(1-p_{n}\right) e^{-\mu(t-s)} S^{*}(s) \int_{0}^{\infty} \bar{i}(a, t) d a d s .
\end{aligned}
$$

Since Theorem 3 and Equation 25, $\lim _{t \rightarrow \infty} s(t)=0$, if $s_{0}$ are small enough. From Equation 20 and the definition of $R_{0}$, we get:

$$
\begin{aligned}
R_{0}^{1} & =S^{*}(T) \int_{0}^{\infty} \beta(a) \int_{0}^{a} e^{-\mu(a-s)-\int_{0}^{a} \alpha(\xi) d \xi} d s d a \\
& =S^{*}(T) \int_{0}^{\infty} \beta(a) \int_{0}^{a} K_{0}(a) e^{\mu s} d s d a \\
& =\frac{S^{*}(T)}{\mu} \int_{0}^{\infty} \beta(a) K_{0}(a)\left(e^{\mu a}-1\right) d a, \\
& >S^{*}(T) \int_{0}^{\infty} \beta(a) K_{0}(a) d a=R_{0} .
\end{aligned}
$$

From Theorems 2 and 3, we lead to:

\section{Theorem 4}

If $\beta(\tau), \alpha(\tau) \in L^{\infty}[0, \infty)$, and $R_{0}^{1}<1$, then the system of Equation 10, that is the system of Equation 1 is globally 


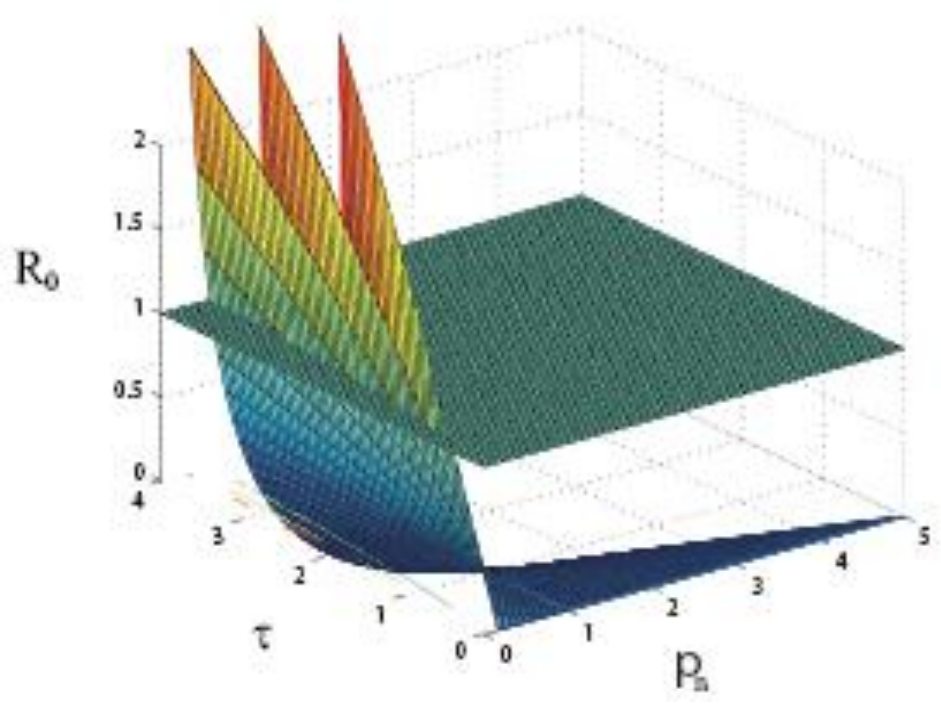

Figure 1. Impulsive persuasion factor in disease eradication.

asymptotically stable at $\left(S^{*}(t), 0,0\right)$.

\section{DISCUSSION}

In this paper, we discuss an HIV/AIDS model with impulse persuasion and infection age and get conditions that lead to the theoretical eradication of HIV/AIDS. The results of this theoretical study are instructive to HIV. We have shown that the impulsive persuasion is a very important factor in disease eradication (Figure 1). From Theorem 4, we know the stability of the infection-free equilibrium $E^{*}$ should depend on $p_{n}$ and $\tau$. Since $R_{0}=S^{*}(T) \int_{0}^{\infty} \beta(a) K_{0}(a) d a$, and

$S^{*}(T)=\frac{\Lambda}{\mu} \frac{e^{-\mu \tau}\left(1-e^{-\mu q \tau}\right)}{1-\prod_{1 \leq k \leq q}\left(1-p_{k}\right) e^{-\mu q \tau}}+\frac{\Lambda}{\mu}\left(1-e^{-\mu \tau}\right), \quad$ we enforce pulse persuasion rate $p_{n}$ and short inter-pulse period $\tau$ such that $R_{0}<R_{0}^{1}<1$ holds. Consequently, the disease is only theoretically eradicated from the population. Our environment is isolated in our model but in reality it should be diffused from one place to another place. With the economic development, recreation develops very fast which enhance difficulty of persuasion. However, the results of this theoretical study are instructive to the study of other diseases such as HINI, SARS, Hepatitis B. If we persuade the susceptible to adopt safe measures, the infected rate must be reduced. Such as SARS in 2003 in China, we persuaded people that were far from dense crowd and the disease quickly was controlled. Here $R_{0}$ is not a basic reproduction ratio (Heffernan et al., 2005), but is a threshold. Since the model is an impulsive system, it is difficult to estimate basic reproduction ratio. We are difficult to explain the biological meaning with the classical method. In future, we believe effects of movement between places could be very important. This would lead to a mixing system of coupled nonlinear partial differential equations with impulsive differential equations.

The effects of particular people groups such as senorita in recreation grounds, poor men unmarried in remote coteaus are also worthy of study. In addition, we will consider the existence of positive period solution in our model.

\section{ACKNOWLEDGMENTS}

This work is supported by the National Sciences Foundation of China (11071283, 10971178), the Sciences Foundation of Shanxi (2009011005-3) and the Young Sciences Foundation of Shanxi (2011021001-1).

\section{REFERENCES}

Aggarwala B (2006). On Estimating HIV in India, Far East J. Math. Sci., 21(2): 197-210.

AIDS Epidemic Update (2007). UNAIDS, Joint United Nations Programme On HIV/AIDS, 3.

Heffernan JM, Smith RJ, Wahl LM (2005). Perspectives on the basic Reproductive Ratio. J. R. Soc. Interface., 2(4): 281-293.

lannelli M (1995). Mathematical theory of age-structured population dynamics, in Applied Mathematics Monographs 7, comitato Nazionale per le Scienze Matematiche, Consiglio Nazionale delle Ricerche (C.N.R.), Giardini, Pisa.

Krakovska O, Wahl LM (2007). Optimal drug treatment regiments for 
HIV depends on adherence. J. Theor. Biol., 246(3): 499-509.

Lakshmikantham V, Bainov DD, Simeonov PS (1989). Theory of Impulsive Differential Equations, vol. 6 of Series in Modern Applied Mathematics, World Scientific, Singapore.

Liu H, Xu H, Yu J (2006). Global asymptotic stability for the SIV epidemic model with impulsive vaccination and infection age, J. Biol. Syst., 14(1): 43-51.

Liu HL, Yu JY, Zhu GT (2008). Global behaviour of an age-infectionstructured HIV model with impulsive drug-treatment strategy. J. Theor. Biol., 253(4): 749-754.

Liu. MX, Jin Z (2006), The effect of Impulsive Control Strategy in HIV/AIDS Model. Dynam. Continuous, Discrete Impulsive Syst., 13: 706-713.

Rahman MS, Rahman ML (2007). Media and education play a tremedous role in mounting AIDS awareness among married couples in Bangladesh, AIDS Res. Therapy, 4: 10-17.

Smith RJ, Wahl LM (2004). Distinct effects of protease and reverse transcriptase inhibition in an immunological model of HIV-1 infection with impulsive drug effects. Bullet. Math. Biol., 66(5): 1259-1283.

Smith RJ, Wahl LM (2005). Drug resistance in an immunological model of HIV-1 infection with impulsive drug effects. Bullet. Math. Biol., 67(4):783-831.
State Council AIDS Working Committee office, UN Theme Group on HIV/AIDS in China. A Joint Assessement of HIV/AIDS Prevention, Treatment and Care in China. Beijing: China Ministry of Health: December 1, 2007.

Thripathi A, Nareshi R, Sharma D (2007) Modeling the effect of screening of unaware infectives on the spread of HIV infection, Appl. Math. Comp., 184: 1053-1068.

Webb GF (1985). Theory of Nonlinear Age-Dependent Population Dynamics, Dekker, New York. 